\title{
Generation of induced pluripotent stem cells from human $\beta$-thalassemia fibroblast cells
}

\author{
Cell Research (2009) 19:1120-1123. doi: 10.1038/cr.2009.100; published online 18 August 2009
}

\section{Dear Editor,}

Induced pluripotent stem (iPS) cells have recently been generated by directly introducing several transcription factors into differentiated human somatic cells, and these iPS cells show great similarities to embryo-derived ES cells [1-3]. Moreover, patient-specific iPS cells have recently been generated, and these studies provided hopes for patients with genetic and degenerative diseases $[4,5]$. $\beta$-thalassemia is an inherited blood disorder that is characterized by reduced synthesis of hemoglobin subunit beta (hemoglobin $\beta$-chain). Individuals with thalassemia major (also called Cooley's anemia) have severe anemia and hepatosplenomegaly; without treatment, affected children have severe failure to thrive and a shortened life expectancy. Even with transfusion and chelation therapy treatments, the life span of patients with thalassemia major can only be extended for a limited time. More importantly, $\beta$-thalassemia patients are widely distributed throughout the southern part of China, and this genetically inherited disease has threatened millions of people's lives for decades with no effective treatment available. Here, we report that we have successfully generated $\beta$-thalassemia-specific iPS cells, which may pave the way to optimize life span-extending treatments for patients with $\beta$-thalassemia major.

We used a previously published protocol [6] to generate $\beta$-thalassemia patient-specific iPS cells. Retroviruses containing human OCT3/4, SOX2, KLF4, and c-MYC were introduced into fibroblast cells derived from a homozygous $\beta$-thalassemia individual. To monitor infection efficiency, a GFP-expressing plasmid pMXs-GFP was used as a control. The single-gene infection efficiency reached approximately $80 \%-90 \%$, as determined by GFP expression. Six days after transduction, the cells were harvested by trypsinization and plated onto mitomycin C-treated MEF feeder cells at a density of $1 \times 10^{5}$ cells per 100-mm dish. Two days later, the medium was replaced with the one typically used for human ES (hES) cell culture that was supplemented with $10 \mathrm{ng} / \mathrm{ml}$ basic fibroblast growth factor.
Approximately 2 weeks after the transduced cells were plated on the feeder cells, two kinds of colonies appeared. Some were granulated colonies that were not similar in morphology to hES cells, whereas the others closely resembled hES cell colonies. These colonies exhibited a flat and tightly packed morphology with sharp edges, and had a high nucleus/cytoplasm ratio and large nucleoli. From each plate, approximately 10 to $50 \mathrm{hES}$ like colonies were obtained, and these colonies were individually isolated and mechanically passaged around 25 days after infection. These cells were passaged every 3-5 days, and they displayed morphologies and growth rates that are similar to those of hES cells (Figure 1A). Herein, we refer to these cells as $\beta$-thalassemia iPS cells. Two patient-specific iPS cell lines were derived, and both showed a normal diploid karyotype (46, XY) (Figure 1B). Genomic sequencing results indicate that the $\beta$-thalassemia iPS cells contain two mutation sites at the Hbb gene, a pattern identical to the parental $\beta$-thalassemia fibroblast cells used for iPS induction (Figure 1C). Short tandem repeat experiments also confirmed that the patterns of the $\beta$-thalassemia iPS cells are completely identical to that of the parental fibroblasts (Supplementary information, Figure S1). Therefore, the $\beta$-thalassemia iPS cells are derivatives of the parental ק41/42-17 fibroblasts and not from contamination with other cell types. Southern blot analysis with both an OCT3/4 cDNA probe and a SOX2 cDNA probe revealed that there are two retroviral integration sites of each in $\beta$-thalassemia iPS cells (Supplementary information, Figure S2).

To validate the characteristics of $\beta$-thalassemia iPS cells, the expression of undifferentiated ES cell marker genes was tested by RT-PCR (Supplementary information, Figure S3). The expression of OCT3/4, SOX2, NANOG, and hES cell-specific surface antigens, including TRA-1-60 and TRA-1-81, was detected by immunocytochemistry staining, whereas SSEA-1 was not detected in the $\beta$-thalassemia iPS cells (Figure 1D). Meanwhile, these two $\beta$-thalassemia iPS cell lines were positive for alkaline phosphatase (AP) staining (Supple- 


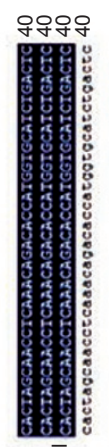

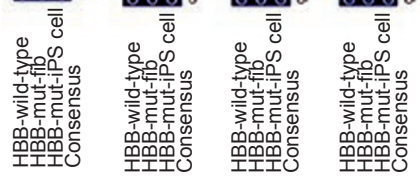

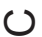
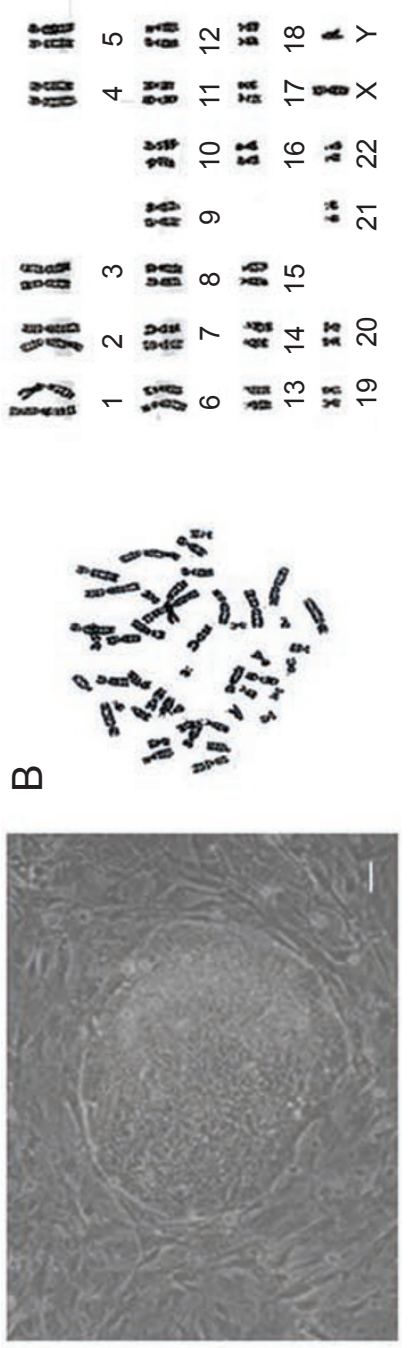

$\varangle$
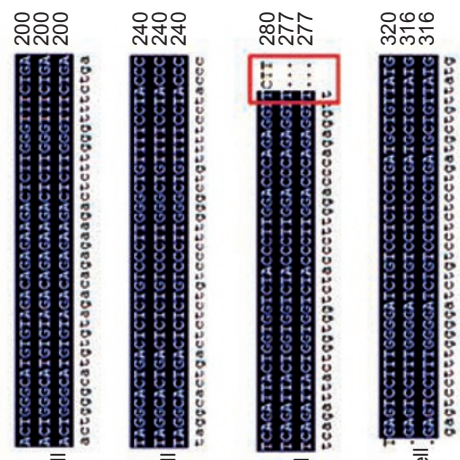

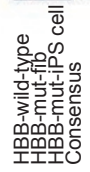

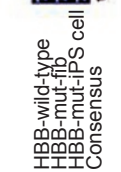

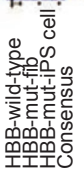

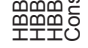
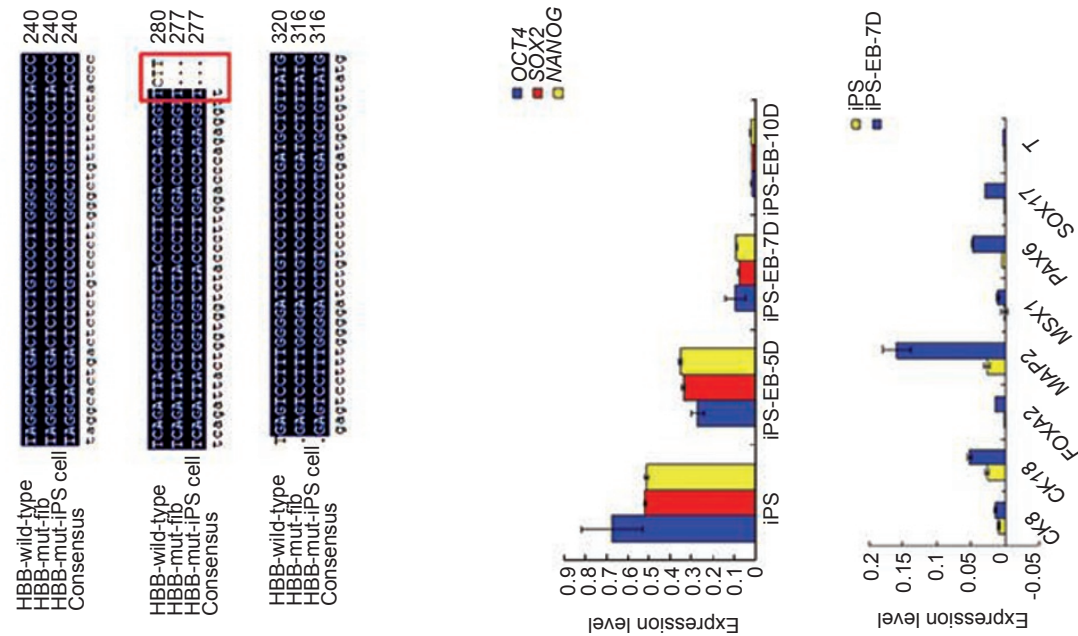

I

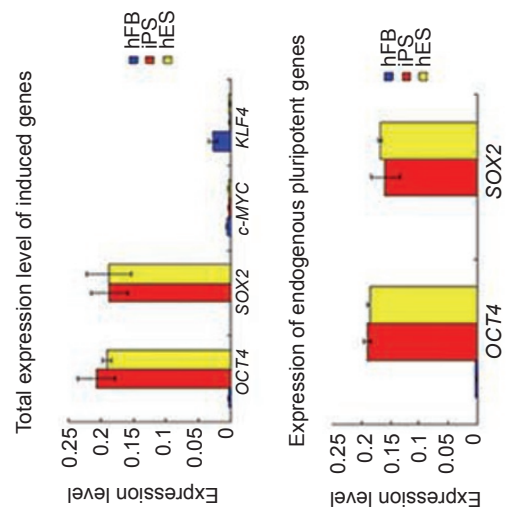

Ш

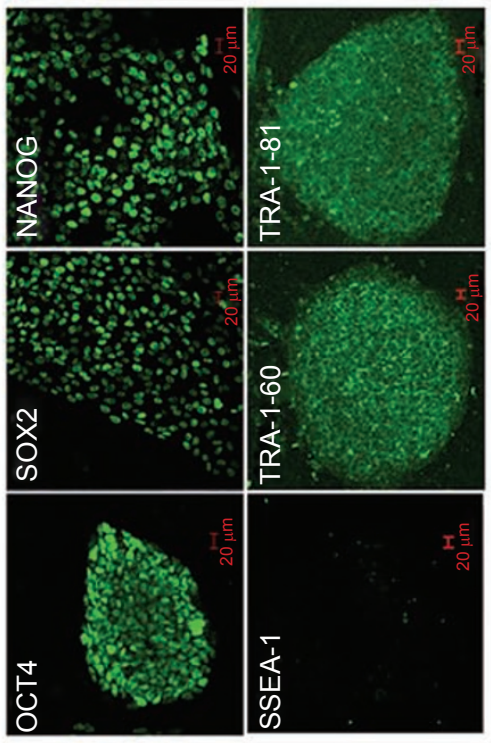

()
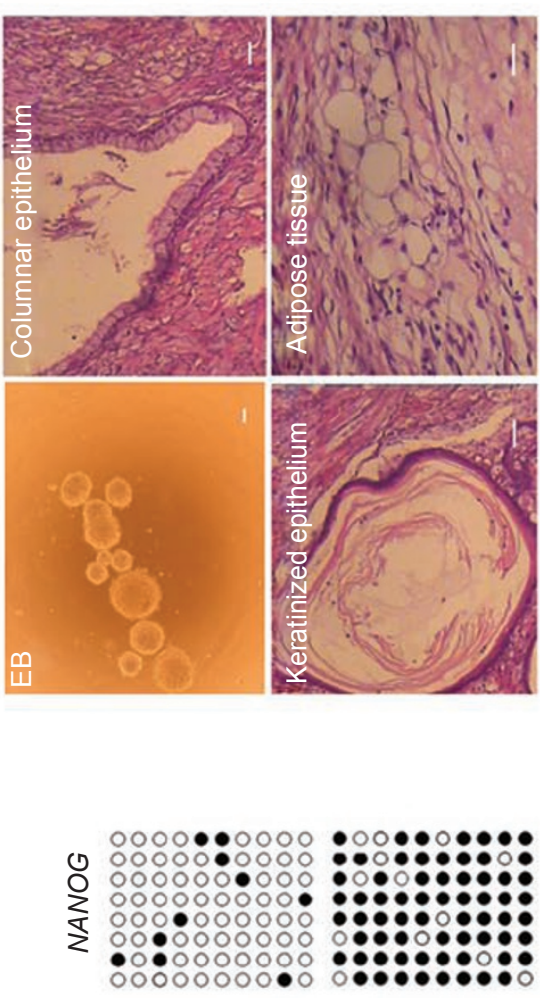

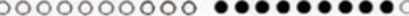
$000000000 \bullet \bullet \bullet \bullet \bullet: \bullet \bullet$

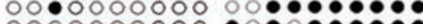
+ 4 U ०000000000 $\bullet \bullet \circ \circ \circ \circ \circ \circ \circ$

Sd!

8.14 
mentary information, Figure S4). Quantitative PCR results revealed that total and endogenous expression levels of OCT3/4 and SOX2 are comparable to that in ES cells, indicating retroviral silencing (Figure 1E).

To investigate the epigenetic status of the $\beta$-thalassemia iPS cells, bisulfite genomic sequencing was used to analyze the methylation status of cytosine guanine dinucleotides $(\mathrm{CpG})$ in the promoter regions of pluripotent-associated genes, such as OCT3/4 and NANOG. The results revealed that they are highly unmethylated, similar to hES cells, whereas the same regions are highly methylated in $\beta 41 / 42-17$ fibroblast cells (Figure 1F).

To determine the differentiation ability of $\beta$-thalassemia iPS cells in vitro and in vivo, both floating cultivation of embryoid bodies (EBs) and teratoma assays were performed. Eight days after the suspension culture was initiated, the iPS cells effectively formed ball-shaped structures, at which time the EBs were transferred onto gelatin-coated plates and culturing continued for another 8 days (Figure $1 \mathrm{G}$ ). The attached cells showed various types of morphologies. Quantitative PCR showed that the expression levels of OCT3/4, SOX2, and NANOG were markedly decreased (Figure $1 \mathrm{H}$ ). In contrast, expression of differentiation-related genes, including cytokeratin 8 and 18 (endoderm), forkhead box A2 (FOXA2, endoderm), microtubule-associated protein 2 (MAP2, ectoderm), Msh homeobox 1 (MSX1, mesoderm), paired box 6 (PAX6, ectoderm), and Brachyury (T, mesoderm), were all significantly upregulated in the differentiated iPS cells (Figure 1H), which was further confirmed by RT-PCR (Supplementary information, Figure S5). In vivo differentiation showed that teratomas were formed 9 weeks after injection; histological examination showed that the tumors contained various tissues of all three germ layers (Figure 1G).

Previous studies have shown that histone deacetylase inhibitor could improve the efficiency of reprogramming mediated either by somatic cell nuclear transfer or by introduction of defined factors. We asked whether VPA treatment would also improve reprogramming efficiency in generation of $\beta$-thalassemia iPS cells. Using AP staining, we counted the numbers of AP-positive colonies in $100-\mathrm{mm}$ dishes of transduced $\beta$-thalassemia fibroblast cells treated with or without VPA, and found that VPA treatment could improve reprogramming efficiency by three- to fourfold (Supplementary information, Figure S6).

Several recently published reports have demonstrated that autologous iPS cells can be generated from patients with degenerative diseases or genetically inherited diseases, such as amyotrophic lateral sclerosis, spinal muscular atrophy, and Parkinson's disease [4, 7, 8]. A more recent published study has demonstrated that Fanconi iPS cells could be generated from disease-corrected patient fibroblast cells and differentiated into hematopoietic progenitors [9]. A mouse model established by Jaenisch's lab clearly indicates that genetically corrected iPS cells, which were derived from a mouse with sickle cell anemia, could rescue the anemia phenotype after transplantation into the bone marrow [10]. Since the clinical application of iPS cells for transplantation therapy is in progress, more studies are necessary to optimize the generation, genetic modification and directed differentiation of iPS cells before the clinical application of iPS cells can be finally realized.

In conclusion, the results of this study clearly demonstrate that $\beta$-thalassemia patient-specific autologous

Figure 1 Generation of $\beta$-thalassemia iPS cells from skin fibroblast cells. (A) Morphology of $\beta$-thalassemia iPS cells at passage 20; scale bar $50 \mu \mathrm{m}$. (B) The karyotype of $\beta$-thalassemia iPS cells is $46, \mathrm{XY}$. (C) Genomic sequencing of Hbb gene showed that the $\beta$-thalassemia iPS cells contain two mutation sites at this gene, a pattern identical to the parental $\beta$-thalassemia fibroblast cells used for iPS induction. (D) Immunostaining of $\beta$-thalassemia iPS cells. $\beta$-Thalassemia iPS cells are positive for pluripotent genes OCT3/4, SOX2, and NANOG, and also express the cell surface markers, TRA-1-60 and TRA-1-81, but not SSEA-1; scale bar $20 \mu \mathrm{m}$. (E) Quantitative PCR results show that the total expression level of OCT3/4, SOX2, c-MYC, and KLF4 in $\beta$-thalassemia iPS cells is similar to that in hES cells (upper panel), and the endogenous expression of OCT3/4 and SOX2 in $\beta$-thalassemia iPS cells is similar to that in hES cells (lower panel). Transcript levels were normalized to GAPDH levels. Error bars represent S.D. $(n=3)$. (F) Bisulfite sequencing analysis of OCT3/4 and NANOG promoters in $\beta$-thalassemia iPS cells and skin fibroblast cells. Each horizontal row of circles represents an individual sequencing reaction for a given amplification. Empty circles represent unmethylated CpG dinucleotides; filled circles represent methylated CpG dinucleotides. (G) In vitro differentiation of $\beta$-thalassemia iPS cells to form embryoid bodies (EBs) (for 8 days in suspension cultivation), scale bar $=100 \mu \mathrm{m}$; and in vivo differentiation of $\beta$-thalassemia iPS cells. Teratomas, which formed 8-10 weeks after injection of the iPS cells, contained tissues from all three germ layers, including keratinized epithelium, adipose tissue and columnar epithelium $(\times 20)$. (H) Quantitative PCR revealed that the expression levels of OCT3/4, SOX2, and NANOG are markedly decreased in iPS-derived EB cells (upper panel), while the expression levels of differentiated related genes are markedly increased in iPS EB cells, including CK 8 and 18 (endoderm), FOXA2 (endoderm), MAP2 (ectoderm), MSX1 (mesoderm), PAX6 (ectoderm), and T (mesoderm) (lower panel). Transcript levels were normalized to GAPDH levels. Error bars represent S.D. $(n=3)$. 
iPS cells can be generated by transduction of four transcription factors into differentiated somatic cells. These cells have the potential to be used in future cell transplantation-based stem cell therapies for patients with $\beta$-thalassemia major, which may lead to the cure of the disease.

\section{Acknowledgments}

Shaorong Gao was supported by the Hi-Tech Research and Development Program of China (863) (2008AA022311 and 2006AA02A101). Xiaofang Sun was supported by grants from the Guangzhou City Science and Technology Administration (2006Z1E0021 and 2008A1-E4011-3) and the Guangdong Province Science and Technology Administration (2007A032100003). We thank the lab members for their helpful comments on the manuscript.

Yixuan Wang ${ }^{2,3, *}$, Yonghua Jiang ${ }^{1,3, *}$, Sheng $\mathrm{Liu}^{3}, \mathrm{Xi}-$ aofang Sun $^{1}$, Shaorong $\mathrm{Gao}^{3}$

${ }^{1}$ Institute of Gynecology and Obstetrics, the Third Affiliated Hospital of Guangzhou Medical College, Guangzhou, China; ${ }^{2}$ Graduate Program in Chinese Academy of Medical Sciences and Peking Union Medical College, Beijing, China; ${ }^{3}$ National Institute of Biological Sciences, Beijing, China

*These two authors contributed equally to this work. Correspondence: Shaorong Gao ${ }^{\mathrm{a}}$, Xiaofang Sun $^{\mathrm{b}}$

${ }^{a}$ Tel: +86-10-80728967; Fax: +86-10-80727535

${ }^{\mathrm{b}}$ Tel: +86-20-81292202; Fax: +86-20-81292013

${ }^{a}$ E-mail: gaoshaorong@nibs.ac.cn

bE-mail: xiaofangsun@hotmail.com

\section{References}

1 Takahashi K, Tanabe K, Ohnuki M, et al. Induction of pluripotent stem cells from adult human fibroblasts by defined factors. Cell 2007; 131:861-872.

2 Yu J, Vodyanik MA, Smuga-Otto K, et al. Induced pluripotent stem cell lines derived from human somatic cells. Science 2007; 318:1917-1920.

3 Park IH, Zhao R, West JA, et al. Reprogramming of human somatic cells to pluripotency with defined factors. Nature 2008; 451:141-146.

4 Dimos JT, Rodolfa KT, Niakan KK, et al. Induced pluripotent stem cells generated from patients with ALS can be differentiated into motor neurons. Science 2008; 321:1218-1221.

5 Park IH, Arora N, Huo H, et al. Disease-specific induced pluripotent stem cells. Cell 2008; 134:877-886.

6 Takahashi K, Okita K, Nakagawa M, Yamanaka S. Induction of pluripotent stem cells from fibroblast cultures. Nat Protoc 2007; 2:3081-3089.

7 Ebert AD, Yu J, Rose Jr FF, et al. Induced pluripotent stem cells from a spinal muscular atrophy patient. Nature 2009; 457:277-280.

8 Soldner F, Hockemeyer D, Beard C, et al. Parkinson's disease patient-derived induced pluripotent stem cells free of viral reprogramming factors. Cell 2009; 136:964-977.

9 Raya A, Rodriguez-Piza I, Guenechea G, et al. Diseasecorrected haematopoietic progenitors from Fanconi anaemia induced pluripotent stem cells. Nature 2009; 460:53-59.

10 Hanna J, Wernig M, Markoulaki S, et al. Treatment of sickle cell anemia mouse model with iPS cells generated from autologous skin. Science 2007; 318:1920-1923.

(Supplementary information is linked to the online version of the paper on the Cell Research website.) 